\title{
Personality psychology: Trends and developments
}

\author{
ALOIS ANGLEITNER \\ Universität Bielefeld, FRG
}

\section{INTRODUCTION}

In the development of science, there are sometimes critical junctures from which new developments arise. It seems to me that personality psychology is now at such a stage. One important development is that the disputes among factor analytically oriented researchers about the right number of dimensions for the classification of individual differences seem to be resolved. The Big Five are now consensually acknowledged. Furthermore, the trait approach has been revived after the attack by Mischel and others. Of course, new conceptualizations such as goal-directed, life-style, or motivationally oriented approaches have been introduced or reinvented. And personality psychologists have turned their attention to more process-orientated approaches, such as how people exhibit behaviours and what the functions of these behaviours are. A rich body of research in this direction is being generated by social psychologists who seem to have especially strong interests in the self domain. Temperament, as a broad reference category for early developing, biologically based personality traits, is also attracting more and more researchers.

To underpin trends and developments, I have counted the journal articles cited in Psychological Abstracts (Psychinfo database) for the following three time intervals: 1975-1979, 1980-1984, and 1985-1989. The total number of publications for these three periods rose steadily from 133238 in the first period to 142199 in the second, and 167869 in the third. The publication rates for selected topics in personality are given in Table 1.

To allow a more detailed evaluation of the publication rates in special topics in personality, I calculated the percentages of these rates taking the overall publications per time interval as the baseline. This calculation allows us to see if some topics show a larger or a smaller increase. Figure 1 may inform us about the attractiveness of special topics in our field.

The self is the leading domain, followed by the personality and the assessment domains.

I will now try to give an overview on trends and developments, some comments, and personal evaluations. Furthermore, I will try to point out possible sidetracks,

This article is based on the Presidential Address at the 5th EAPP-conference, Rome/Ariccia-Genzano, June 12-15, 1990.

Correspondence concerning this article should be addressed to Alois Angleitner, Abteilung Psychologie der Universität Bielefeld, Postfach 8640, D-4800 Bielefeld 1, FRG. 
Table 1. Publication rates on special topics in personality in three selected time intervals

\begin{tabular}{lccc}
\hline Topic & \multicolumn{3}{c}{ Time interval } \\
\cline { 2 - 4 } & $1975-1979$ & $1980-1984$ & $1985-1989$ \\
\hline (1) Total psychological publications & 133238 & 142199 & 167869 \\
(2) Psychological publications on special & & & \\
topics in personality [(3) to (11)] & 18724 & 25679 & 28392 \\
(3) Self [without (4)] & 8616 & 12038 & 13177 \\
(4) Personality measurement, personality & & & \\
questionnaire, personality inventory, & & & \\
self-report, self-rating, & 2517 & 3629 & 4555 \\
self-assessment & 1890 & 2698 & 2761 \\
(5) Trait(s) & -1 & 14 \\
(6) Big Five/Five-factor model & 4803 & 5639 & 5856 \\
(7) Personality [without (4)] & 173 & 367 & 463 \\
(8) Temperament & 682 & 1210 & 1458 \\
(9) Psychoanalysis & 26 & 63 & 73 \\
(10) Sociobiology & 17 & 34 & 35 \\
(11) Behaviour genetics & & & \\
\hline
\end{tabular}

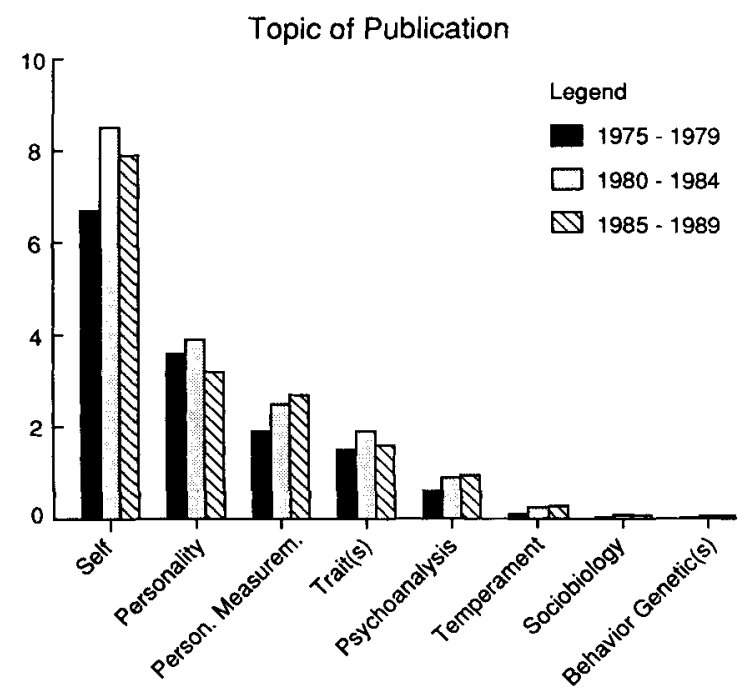

Figure 1. Percentages of publication rates in selected topics taking the overall publications per time interval as the baseline

and neglected and hidden assumptions, of which we should be aware. I will organize this presentation according to the following five topics:

1. What are the most promising basic units for measuring and conceptualizing personality?

2. Traits are alive and well.

3. The promise of the factor analytic strategy.

4. What kinds of personality data should we collect, and how should we conceptualize their convergence? 
5. How to create the right measurement devices for our concepts.

\section{WHAT ARE THE MOST PROMISING BASIC UNITS FOR MEASURING AND CONCEPTUALIZING PERSONALITY?}

The most prominent units in the last 50 years have been traits and motives (needs). For many prominent personality psychologists, personality psychology is synonymous with the study of traits (see, for example, Guilford and Allport). However, there are also scientists whom we may call cognitively, phenomenologically, or psychodynamically oriented. For these colleagues the basic units are, for instance, personal constructs, motives, or the two concepts recently introduced by Little (1983) (i.e. 'personal projects') and Emmons (1986) (i.e. 'personal strivings - or goals'). These last two concepts show some similarity to Thomae's conceptualizations, which he calls Daseinsthemen: themes that dominate a person's life history. But the concepts proposed by Little and Emmons are not so far-reaching. Other researchers have invoked the concepts of life tasks, with which they try to identify the important tasks in the individual life course. Different, mostly cognitively tuned strategies are proposed for dealing with such tasks. I should like to add that Thomae's conceptualizations of Daseinstechniken published in 1960 are very similar to recent formulations.

A careful reading of Murray (1938) should reveal that some of these newly introduced units are only reformulations or refinements of already available thoughts. The newest invention of 'conditional dispositions' by Wright and Mischel (1987) seems to be a rediscovery of the concept of press and its interaction with traits. The authors concentrate on the environmental conditions that are predictive of certain behavioural responses.

The motive-oriented approaches share intentionality and goal orientation. Whereas the various motive conceptions concentrate on the different goals of individuals, we may add the views of Hettema (1979) and Buss (1984, 1991), who point to tactics and strategies as units in an evolutionary frame of reference (enhancing the reproductive success of individuals). There is some ambiguity in separating traits from needs. Remember that many of the psychological needs postulated by Murray (1938) are seen by other scientists as belonging to the trait domain. We may conclude that each trait explanation can be traced back to assumed intentional states and motives. With the exception of some stylistic traits, there is always the possibility that one trait may be explained by different underlying motivations; for example, people may be friendly, because they think that they are more liked or promoted through friendliness. However, psychologists have learned that there are traits rooted in biological processes or the genetic endowment. I would say that the temperament traits belong to this last group. The most intriguing question is the following: Why do people possess these traits and what is their function?

This diversity in the choice of elementary units in describing personality is only enriching to a limited degree. Traits and needs are, to some extent, losing their prominence as units in personality research. However, we should not overvalue newness. It seems to me that we are producing theories, mini-theories, and models without doing our best to try to interconnect these approaches, to search for sufficient agreement, and to strive for the goal of scientific truth. The last is largely forgotten.

Coming back to the question of what are the most promising units for measuring 
and conceptualizing personality, the answer is many. However, I still think that the trait conception is especially promising.

\section{TRAITS ARE ALIVE AND WELL}

In his well-known critical monograph on the weaknesses of the classical trait approach, Mischel (1968) argues that traits may be illusory constructs in the eyes of observers. They have nothing or almost nothing to do with generalized behavioural patterns in the person observed.

The most central points of this criticism refer to the supposed behavioural consistency across different situations as well as to the assumption of the systematic covariation of behaviour with the traits in question. Shweder and D'Andrade's systematic distortion hypothesis (1980) takes a similar viewpoint in arguing that memorybased trait ratings are not based on behavioural co-occurrences but rather on implicit assumptions about what goes with what semantically. In 1977, Shweder wrote: 'The behavioural traits that play such an important role in our everyday descriptions of personality are not 'out there' waiting to be discovered, but rather are the creations of the magical mind' (p. 639). From today's perspective, and many studies, I conclude that there is enough evidence to accept the trait approach. Personality coefficients well above the 0.30 barrier have been reported and behavioural consistency over different relevant situations has been documented. One may conclude, however, that Mischel's attack has been very helpful in clarifying the concept of traits and the problems underlying their convergent and discriminant validity. I remember that my first reaction while reading Mischel's monograph was that I wanted to know how he deals with Cattell's behaviour equation that predicts behaviour from a linear combination of several traits which are weighted by their situational relevance (Cattell, 1946, pp. $558 \mathrm{ff}$.). But Mischel never referred to Cattell's equation. The traits selected by Mischel as well as the situations and behaviours that he offered as examples refuting the trait concept and its predictive power seemed to me somewhat odd and not very convincing. However, it is to Mischel's credit that he has brought us back to the study of behaviour, on which personality psychology used to be focused in the 1920 s and 1930 s when one recalls the studies in real-life settings undertaken by Dudycha (1936) on punctuality, Hartshorne and May (1928) on honesty, Newcomb (1929) on extraversion, and Allport and Vernon (1933) on expressive behaviour. It is probably also due to Mischel's influence that personality psychologists are currently more aware of the necessity of studying the relation between personality variables and real-life outcomes in work, academic, or marital settings. After hundreds of studies reporting correlations or factor analytic results of personality inventory scales, it it refreshing that, as Funder (1989) puts it, 'personality psychologists are finally breaking free of their fascination, indeed obsession, with questionnaires and the mathematical tricks you can play with them' (p. 210).

Coming back to the systematic distortion hypothesis: This hypothesis states that there is low structural correspondence between on-line coded behaviour frequencies and memory based ratings, and high structural correspondence between semantic relations and correlations among memory-based ratings. Studies by Borkenau (1986a, b) and others have clearly demonstrated that this hypothesis is an artifact that owes its existence to a classification system that forces observers to classify observations 
into only one category. If you allow the observers to encode the different behaviours into more than one single category, the distortion effect diminishes. It is strange and somewhat counter intuitive that this distortion hypothesis gained so much attention and popularity. Not one of us would accept that a particular behavioural manifestation relates to only one trait, disposition, or need. Borkenau $(1986 \mathrm{a}, \mathrm{b})$ formulated the systematic overlap hypothesis stating that the more that two behaviour-descriptive terms are similar in meaning, the more they refer to overlapping behavioural act universes.

\section{THE PROMISE OF THE FACTOR ANALYTIC STRATEGY}

For more than 50 years, personality psychologists have attempted to find the basis dimensions of individual differences. The problem with this approach has been that there are as many personality factor models as there are researchers. For example, Cattell (1957) suggests 16-23 normal personality factors with an additional 12 clinical personality factors for the questionnaire domain and 21 objective test factors. Eysenck (1986) postulates the superfactors E, N, and P. Comrey (1970) adheres to 8; Gough suggests 20; Guilford, Zimmerman and Guilford (1976) introduce 10 factors; while Browne and Howarth (1977) suggest 11 stable personality factors. Reasons for these discrepancies lie in the differences among the factor analytic methods, in the different rotational procedures employed, in the underlying sample characteristics (normal vs. abnormal samples), and in the selection of variables. However, the most critical feature seems to be that, with the exception of Cattell, there was no reflection on what should be considered as a comprehensive data set representing the universe of the individual differences under study. The sedimentation hypothesis proposed by Cattell is a rationale for defining representativeness: those individual differences that are most significant in the daily transactions between persons became encoded into their language. This means that one should start to define the personality sphere by trait taxonomic studies of different languages. It is part of the assumption that no large area is neglected in the vocabulary, and that all dimensions of personality receive some representation. Such trait taxonomic work was started by the German psychologist Baumgarten (1933). However, Allport and Odbert's (1936) work received much more attention. More recent studies have been done by Goldberg (1981, 1982) and Norman (1967) for American English; by Hofstee (1977) and the Groningen group for the Dutch language (Brokken, 1978); and by John, Angleitner and Ostendorf (1988) for the German language. Studies starting with personality descriptive adjectives in self- and peer-ratings have now reached consensus about the Big Five factors of personality in different languages such as English, German, and Japanese. And in several studies, it has been shown that the personality questionnaires constructed by Cattell, Eysenck and Guilford can be integrated into the Big Five model (McCrae, 1989). The same applies to other instruments based on very different theoretical assumptions, like Murray's needs, Block's psychodynamic descriptors, and Jung's typological model. It may be noted that there is some discussion concerning the interpretation of such factors as Neuroticism, Extraversion, Agreeableness, Conscientiousness, and Intellect. In particular, the meaning of the fifth factor described as Intellect, Culture, or Openness is unclear. In our German trait taxonomy, we selected those trait adjectives that a majority of judges judged to be personality- 
descriptive. Some of these 430 adjectives were members of one of the two classes (1) temperament and character terms, or (2) ability terms. Whereas factor analyses of the joint sets of adjectives clearly demonstrate robust five-factor solutions, the separate factorization of the temperament and character terms reveals only a fourfactor solution in which no Culture, Openness, or Intellect factor appears. These results strengthen the interpretation of the fifth factor as intellect.

In agreement with Strelau (1987), I suggest a distinction between temperament and personality. Temperament refers to early developing, stable personality traits that relate to more stylistic behavioural tendencies based on the constitutional or biologically determined make-up of individuals. In content analyses of items from several temperament inventories for adults, my colleague Riemann and I found a greater percentage of items referring to overt behavioural reactions than could be found in personality questionnaires (Angleitner and Riemann, 1991). In personality inventories, there is a greater diversity of content, such as covert behavioural reactions, trait attributions, wishes and interests, attitudes, symptoms, and so forth, as well as overt behavioural reactions. This refers to the broader scope of personality that also includes values, attitudes, and interests. I see the first four factors of the Big Five as primarily temperamental dimensions.

However, the greatest need is for studies that would document (1) the replicability of the Big Five in culturally more distant ethnic and language groups; (2) the biological background of these factors as well as their genetic origin; (3) the stability of the Big Five during the life course; (4) the predictive power of these factors for behavior in and outside the laboratory; and (5) the primary factor structure of the Big Five factors.

Such studies would provide arguments against the viewpoint of those in our field who still believe that the Big Five are primarily factors of the language that we use for describing personality.

After 50 years, factor analysis seems to have fulfilled its early promise by producing a consensual set of personality factors. It may be kept in mind that the Big Five represent the broadest level in the hierarchical conception of traits and are thus comparable to such concepts as 'animal' or 'plant' in the world of natural objects. They are useful for rough distinctions and for classifications at very high levels of abstraction. Nevertheless, the Big Five should not be seen as a substitute for considering the more specific traits.

\section{WHAT KINDS OF PERSONALITY DATA SHOULD WE COLLECT AND HOW SHOULD WE CONCEPTUALIZE THEIR CONVERGENCE?}

Apart from Cattell $(1946,1957)$ and Kelly (1955), personality theorists have not connected their data gathering with their approaches on a theoretical level. The favourite measurement procedures are inventories. The convergence with different data sources including laboratory tests, peer ratings, and so forth is treated as a validity issue. However, the search for convergent validity is more dominant, and the issue of discriminant validity is not considered as equally important.

If one believes in source traits, as does Cattell, or in basic needs, like Murray, the conclusion is straightforward: these traits or needs should be detectable in the life-record (L), questionnaire (Q), and objective (T) test data domains. Summarizing 
the results, we may state that there is convergence in primary and secondary factors between the L- and Q-domains, but almost no convergence with the T-data. In Cattell's view, the correspondence should be conceived in the following way: secondary factors of L-and Q-data correspond to the primary factors of the T-data level.

The reasons for this hypothesis are formulated in Cattell's $(1968,1977)$ perturbation theory. In this theory, the two most important perturbation sources are:

1. Instrument factors. These are factors that are connected with the special make-up of the instruments; for instance, questionnaires have similar structures; and

2. Density of the representation of variables in the sense of situation-contingency factors. Q-Data are supposed to possess more generality than T-data, because the $\mathrm{T}$-data are bound to the behaviours elicited in the test situation. You need fewer variables in the Q-data domain compared with the T- (and L-) domain for the reliable assessment of a factor.

From the density argument, however, one may derive an opposite conclusion: $\mathrm{T}$-factors of the secondary order correspond to the primary factors in the L- and Q-domains.

Olweus (1980) points to the basic differences in the data domains. L- and Q-data rely heavily on habitual reactions. T-data are gathered under highly specific conditions and the procedures for gathering them show unsatisfactory reliabilities. Olweus recommends aggregating T-data over repeated measurement occasions.

From the 81 objective-analytic test devices for group testing published by Cattell and Schuerger (1976) in the O-A test battery, about two-thirds are questionnaires. They differ from 'traditional' questionnaires in that they utilize variables like extremity of response, duration of response in answering some attitude items, and so forth.

About 5 per cent of the roughly 400 test devices reported in Cattell's different books may be grouped in the category 'psychophysiological assessment devices', including the famous PGR, pulse rate, heart rate, and so forth. The rest are primarily perceptual tests, mazes, hidden figures, and the like. After studying Cattell, I am surprised that, with the exception of a few devoted scholars, his tremendous effort in collecting as well as constructing objective test devices has had almost no impact on the assessment of personality. I am looking forward to studies relating some of these test devices to temperamental factors, especially to the Big Five, by means of the more adequate design of aggregating objective test scores.

Apart from Cattell, the personality psychologist uses psychophysiological test devices for two purposes: (1) Psychophysiological measures are used as devices for measuring temperamental traits; for instance, the evoked potential measure for augmenting-reducing developed by Buchsbaum (1976), or the Neo-Pavlovian Russian temperament EEG measures. (2) Psychophysiological measures are also used in validity studies for certain psychological concepts like extraversion-introversion, sensation-seeking, neuroticism, anxiety, and so on.

There have been numerous attempts to correlate rather crude single physiological measures with personality variables measured mostly via questionnaires. The accumulated evidence of such enterprises is in no way encouraging. I refer, for example, to a forthcoming paper by Strelau (1991), to Amelang's (1986) disconforming results on Eysenck's hypotheses concerning extraversion-introversion, and to Fahrenberg's (1991) findings on the specificity of psychophysiological data depending on modality, time, and situational circumstances. 
Indeed, a cautionary note may be introduced regarding psychophysiological tests. Inventories and rating scales measuring traits implicitly accept a frequency assumption, which means that an individual's position on variable $X$ is a function of the frequency of the registered behaviour. However, psychophysiological tests generally accept an intensity paradigm, meaning that the intensity of the PGR reaction, and not the frequency, is the critical measure. Usually, personality psychologists do not ask how gregarious you are able to be; they ask how often have you been gregarious in the last few months. In my opinion, the relation of intensity and frequency is not at all clear.

It should be recalled that for measuring states we rely on the intensity paradigm. We may therefore hypothesize that psychophysiological tests show a stronger relation to states than to traits. To my knowledge, this is generally the case.

Should we continue to relate psychophysiological test data to other kinds of data? My answer is a clear yes for the area of temperament and no for the much broader area of personality. But first, the physiological database should be made more reliable, and also different scoring devices should be explored; for instance, the range or the variability of psychophysiological responses over several measurement occasions could be used (see, for example, Fahrenberg's multivariate-multimodality-multioccasion assessment programme). After we have detected replicable physiological patterns, we can consider ways of matching them with known psychological dimensions. New research options will be opened with the development of portable systems for monitoring physiological functions which can be also used for field studies outside the laboratory. We can consider such measures as similar to inventory items and apply item analytic and/or factor analytic strategies to develop composite scores.

\section{HOW TO CREATE THE RIGHT MEASUREMENT DEVICES FOR OUR CONCEPTS}

I will concentrate here on a rational strategy, and I see several solutions that may be viewed as points on a continuum with the poles characterizing the relative contribution of the researcher versus the involvement of the test person.

The most common strategy and one with the lowest representativeness, comprehensiveness, and objectivity is when the researchers themselves create and nominate the behavioural manifestations (sometimes with the help of a glass of wine or through the inspiration of looking up different inventories). This strategy is the most fallible, because of the idiosyncracies involved. Armed with such a list, one starts the empirical study. Based on some overall regularity in behaviour, the researcher will come up with a list of behaviours with which he or she may feel satisfied. However, another researcher may generate a slightly different list. This list also gains some empirical support. The correlation of the different lists, let's say, is 0.50 . This value satisfies our researchers because it tells them what they want to hear. Such a correlation is interpreted as an indication that the concepts show some convergence. On the other hand, it indicates a sufficient divergence to ensure the survival of one's own list in further research. To keep the competing conceptions of, for instance, anxiety, distinguishable, the preferred strategy is to label it with the name of the researcher or the town or university where the researcher is based. This strategy ignores the beliefs and assumptions of the persons taking the test. Because the testees are in 
no way involved in the process of generating the behaviour manifestations that constitute the test items, we should not label a person inconsistent if he or she fails to exhibit behavioural manifestations that are supposed to co-vary. Such a person is only inconsistent with the presupposed hypothesis of the researcher about what goes with what.

A somewhat different, but cumbersome, strategy with a medium level of representativeness, comprehensiveness, and objectivity involves a panel of experts, preferably from different cultures, in the development of a pool of assumed behavioural manifestations for a given trait or concept. After surviving a discussion by experts, such a list may be more representative and exhaustive than a list generated by a single researcher. This strategy is preferable if you want to measure concepts for which lay-persons have no information. We have used this strategy for measuring the Pavlovian concepts, strength of excitation, strength of inhibition, and mobility on the behavioural level (Strelau, Angleitner, Bantelmann and Ruch, 1990).

For the domains of traits, needs, motives, interests, and values, I would suggest that we should already engage our subjects in the nomination of the relevant behavioural examples. In particular, a similar approach to that advocated by the authors of the act frequency approach seems fruitful (Buss and Craik, 1983). In the act-nomination procedure, the researcher tries to identify the behavioural manifestations that can be subsumed for a trait or disposition. Act nominations can occur from direct observation or retrospectively. However, retrospective act nominations have been used most frequently. Subjects are given, for instance, the instruction to think of the three most dominant individuals they know and to write down five specific acts or behaviours that these individuals have performed that reflect or exemplify their dominance. For instance, a typical example of a behavioural act for dominance is 'He/she sets goals for a group.' Such lists of edited and rephrased behavioural acts are then given to judges, who rate their prototypicality for the respective trait. The issue here is whether those acts are good examples of the trait in question. Such act lists have been generated particularly for the interpersonal trait domain. Amelang was also able to apply this strategy successfully for the creativity domain (personal communication). We may conclude that after passing the prototypicality test, such lists containing highly prototypical behavioural manifestations may be regarded as possessing a much higher degree of exhaustiveness and representativeness than the lists generated by the two strategies mentioned above. Exhaustiveness, representativeness, and objectivity would be maximized if one could collect all possible behavioural manifestations that people are able to express in their languages. This, however, would be impossible.

The frequencies of each behaviour description nominated, the number of synonym expressions for a given concept, and the universality of equivalent descriptions for the same concept across languages and cultures would give us some hint about the life importance of these behaviours. However, such an enterprise has not been started until now, and it is questionable whether it will ever be completed. One way out of this somewhat unsatisfactory situation would be to look out for more generalized units of behavioural expressions. This brings us back to traits, dispositions, or needs, which may be seen as convenient summary labels for individual differences in behaviour. It seems to me that we try to move into the behavioural domain because we think that there is much more objectivity and maybe reality involved in having subjects answer an item such as 'likes to speak to other people' 
instead of just asking the subjects or their peers how talkative or silent they are. In adjective ratings, there is no context specification involved. But after having worked extensively with questionnaires for 15 years, I would conclude that it does not matter whether you do or do not specify the context. The subjects themselves make the item understandable by reinterpreting it in such a way that it fits their personal life circumstances and their view of themselves and other persons.

A number of studies have documented that the 0.30 correlation barrier has been broken through. In our multitrait-multimethod studies in validating the German PRF with self and peer ratings on the level of the PRF-scale definitions, we found the following correlations for five different samples: the medians for PRF $x$ self rating ranged from 0.56 to 0.70 ; for self rating $x$ peer rating: $0.35-0.57$; and PRF $\mathrm{x}$ peer rating: $0.36-0.52$ (all values without correction for attenuation) (Ostendorf, Angleitner and Ruch, 1986).

In our German trait taxonomy project, we found the correlations shown in Table 2 among factor scores derived from unipolar, peer-rating scales with the NEO-PI for the Big Five (Angleitner and Ostendorf, 1989).

Table 2. Correlations among factor scores derived from unipolar peer-rating scales and the NEO Personality Inventory

\begin{tabular}{lccccccc}
\hline $\begin{array}{l}\text { Question- } \\
\text { naire and } \\
\text { rating } \\
\text { factors }\end{array}$ & SU & AG & CO & ES & CU & $\begin{array}{c}\text { Mean } \\
\text { values }\end{array}$ \\
\hline $\begin{array}{l}\text { NEO-PI- } \\
\text { VX }\end{array}$ & $0.74^{*}, 0.50$ & $0.72^{*}, 0.47$ & $0.67 *, 0.51$ & $0.67^{*}, 0.41$ & $0.59^{*}, 0.39$ & $0.68,0.46$ \\
$\begin{array}{l}\text { Unipolar } \\
\text { SR }\end{array}$ & 0.73 & 0.58 & 0.72 & 0.66 & 0.56 & 0.66 \\
\hline
\end{tabular}

Note: The first correlation in the NEO-PI-VX row refers to peer-ratings; the second correlation refers to self-ratings. NEO-PI-VX: Validimax-rotated NEO-PI factor scores. Unipolar SR: Self-ratings on 430 unipolar rating scales. $\mathrm{SU}=$ Surgency; $\mathrm{AG}=$ Agreeableness; $\mathrm{CO}=$ Conscientiousness; $\mathrm{ES}=\mathrm{Emo-}$ tional Stability; $\mathrm{CU}=$ Culture.

$* N=110 ; N=394$ (peer-ratings); $N=408$ (self-ratings).

Coming back to personality inventories, I think that we can learn two lessons from our studies dealing with items of personality inventories (Angleitner, John and Löhr, 1986). First, in models about the process of answering inventory items, there is convergence of the proposed three stages in answering a personality test item. These stages are encoding, item-self comparison, and utility control. Our research on the information processing characteristics of personality items, however, revealed that more than 50 per cent of inventory items are difficult to understand, 25 per cent are ambiguous, and about 50 per cent are abstract and value-loaded.

Second, the syntactics of the item surface structure (active, passive, tense, length) as well as aspects of the response process, such as understandable, ambiguous, abstract, not self-referent and evaluative item contents, have been shown to be the most powerful predictors of response stability and item validity.

Our findings imply that the influence of 'formal' item characteristics on the psychometric quality of personality inventories is both systematic and substantial. The surface structure of the item is an essential determinant of the reliability and validity 
of the item responses of our subjects. If you want to construct a personality scale, you are advised, after the generation of the item pool, with the help of experts and your subjects, to spend considerably more time and care in formulating item sentences.

Coming back to the beginning of this contribution, I should like to reconsider the issue of publication rates. We have to face the problem that there is an explosion of psychological knowledge. It seems to me that the current production of psychological knowledge cannot be grasped by a single researcher. This also results in the neglect of psychological literature produced, for example, in the $1950 \mathrm{~s}$ as well as earlier. As a consequence, new approaches may be generated that were already proposed 50 years ago but have since been forgotten. In the computerized age, the psychological literature published before 1970 has not yet been systematically covered. Production leads to further differentiation. It may well be that we will soon have, besides journals of aggressive or creative behaviour, a journal of altruistic behaviour, a journal of bargaining behaviour, and so forth. The huge amount of psychological production calls for more cooperation between researchers to compensate for the limited capacity of the single researcher. Furthermore, more exchange of information between scientists is necessary. In this sense, the activities of the EAPP in organizing workshops and conferences can be expected to further the development of psychological science.

\section{REFERENCES}

Allport, G. W. and Odbert, H. S. (1936). 'Trait-names: a psycho-logical study. Psychological Monographs, 47: No. 211.

Allport, G. W. and Vernon, P. E. (1933). Studies in Expressive Movement, Macmillan, New York.

Amelang, M. (1986). 'Fragebogen-Tests und experimentalpsychologische Variablen als Korrelate der Persönlichkeitsdimensionen Extraversion/Introversion (E/I) und Neurotizismus (N)' [Questionnaire-tests and experimental psychological variables as correlates of the personality traits Extraversion/Introversion (E/I) and Neuroticism (N)]. In: Amelang, M. (Ed), Bericht über den 35. Kongreß der Deutschen Gesellschaft für Psychologie in Heidelberg 1986, Vol. 2, pp. 403-416, Hogrefe, Göttingen, FRG.

Angleitner, A. and Ostendorf, F. (1989). 'Personality factors via self- and peer-ratings based on a representative sample of German trait descriptive terms', Paper presented at the 1st European Congress of Psychology, Amsterdam, The Netherlands, 2-7 July.

Angleitner, A. and Riemann, R. (1991). 'What can we learn from the discussion of personality questionnaires for the construction of temperament inventories?' In: Strelau, J. and Angleitner, A. (Eds), Explorations in Temperament, Plenum Press, New York (in press).

Angleitner, A., John, O. P. and Löhr, F. J. (1986). 'It's what you ask and how you ask it: an itemmetric analysis of personality questionnaires.' In: Angleitner, A. and Wiggins, J. S. (Eds), Personality Assessment via Questionnaires: Current Issues in Theory and Measurement, pp. 61-108, Springer, Heidelberg, FRG.

Baumgarten, F. (1933). 'Die Charaktereigenschaften' [The character traits]. In: Baumgarten, F. (Ed), Beiträge zur Charakter- und Persönlichkeitsforschung [Contributions to Research in Character and Personality], Whole No. I, Francke, Bern, Switzerland.

Borkenau, P. (1986a). 'Systematic distortions in the recognition of trait information'. In: Angleitner, A., Furnham, A. and Van Heck, G. L. (Eds), Personality Psychology in Europe: Current Trends and Controversies, Vol. 2, pp. 177-191, Swets \& Zeitlinger, Lisse, The Netherlands.

Borkenau, P. (1986b). 'Toward an understanding of trait interrelations: acts as instances for several traits', Journal of Personality and Social Psychology, 51: 371-381.

Brokken, F. B. (1978). The Language of Personality, Krips, Meppel, The Netherlands. 
Browne, J. A. and Howarth, E. (1977). 'A comprehensive factor analysis of personality questionnaire items: a test of twenty putative factor hypotheses', Multivariate Behavioral Research, 12: 399-427.

Buchsbaum, M. S. (1976). 'Self-regulation of stimulus intensity: augmenting/reducing and the average evoked response'. In: Schwartz, G. E. and Shapiro, D. (Eds), Consciousness and Self-regulation, Plenum Press, New York.

Buss, D. M. (1984). 'Evolutionary biology and personality psychology: toward a conception of human nature and individual differences', American Psychologist, 39: 1135-1147.

Buss, D. M. (1991). 'Evolutionary personality psychology', Annual Review of Psychology, 42: 459-491.

Buss, D. M. and Craik, K. H. (1983). 'The act frequency approach to personality', Psychological Review, 90: 105-126.

Cattell, R. B. (1946). The Description and Measurement of Personality, Harcourt Brace Jovanovich, New York.

Cattell, R. B. (1957). Personality and Motivation Structure and Measurement, Harcourt Brace Jovanovich, New York.

Cattell, R. B. (1968). 'Trait-view theory of perturbations in ratings and self ratings (L (BR)and Q-data): its application to obtaining pure trait score estimates in questionnaires', Psychological Review, 75: 96-113.

Cattell, R. B. (1977). 'A more sophisticated look at structure: perturbation, sampling, role, and observer trait-view theories'. In: Cattell, R. B. and Dreger, R. M. (Eds), Handbook of Modern Personality Theory, pp. 166-220, Halsted Press, New York.

Cattell, R. B. and Schuerger, J. (1976). The $O-A$ (Objective-Analytic) Personality Battery, Institute for Personality and Ability Testing, Champaign, IL.

Comrey, A. L. (1970). Manual for the Comrey Personality Scales, Educational and Industrial Testing Service, San Diego, CA.

Dudycha, G. J. (1936). 'An objective study of personality research', Archives of Psychology, 29: 1-53.

Emmons, R. A. (1986). 'Personal strivings: an approach to personality and subjective wellbeing', Journal of Personality and Social Psychology, 51: 1058-1068.

Eysenck, H. J. (1986). 'Models and paradigms in personality research'. In: Angleitner, A., Furnham, A. and Van Heck, G. L. (Eds), Personality Psychology in Europe: Current Trends and Controversies, Vol. 2, pp. 213-224, Swets \& Zeitlinger, Lisse, The Netherlands.

Eysenck, H. J. and Eysenck, M. W. (1985). Personality and Individual Differences. A Natural Science Approach, Plenum Press, New York.

Fahrenberg, J. (1991). 'Differential psychophysiology and the diagnosis of temperament'. In: Strelau, J. and Angleitner, A. (Eds), Explorations in Temperament, Plenum Press, New York (in press).

Funder, D. C. (1989). 'Accuracy in personality judgement and the dancing bear'. In: Buss, D. M. and Cantor, N. (Eds), Personality Psychology. Recent Trends and Emerging Directions, pp. 210-223, Springer, New York.

Goldberg, L. R. (1981). 'Language and individual differences: the search for universals in personality lexicons'. In: Wheeler, L. (Ed), Review of Personality and Social Psychology, Vol. 2, pp. 141-165, Sage, Beverly Hills, CA.

Goldberg, L. R. (1982). 'From ace to zombi: some explorations in the language of personality'. In: Spielberger, C. D. and Butcher, J. N. (Eds), Advances in Personality Assessment, Vol. 1, pp. 203-234, Erlbaum, Hillsdale, NJ.

Guilford, J. S., Zimmerman, W. S. and Guilford, J. P. (1976). The Guilford-Zimmerman Temperament Survey Handbook. Twenty-five Years of Research and Application, Edits Publishers, San Diego, CA.

Hartshorne, H. and May, M. A. (1928). Studies in the Nature of Character. Volume I. Studies in Deceit, Macmillan, New York.

Hettema, P. J. (1979). Personality and Adaptation, North-Holland, Amsterdam, The Netherlands.

Hofstee, W. K. B. (1977). 'Preliminary steps in structuring the set of 1204 Dutch personalitydescriptive adjectives', Heymans Bulletins, HB-77-302 IN, University of Groningen, The Netherlands. 
John, O. P., Angleitner, A. and Ostendorf, F. (1988). 'The lexical approach to personality: a historical review of trait taxonomic research', European Journal of Personality, 2: 171-203.

Kelly, G. A. (1955). The Psychology of Personal Constructs, Norton, New York.

Little, B. R. (1983). 'Personal projects: a rationale and method for investigation', Environment and Behavior, 15: 273-309.

McCrae, R. R. (1989). 'Why I advocate the five-factor model: joint factor analyses of the NEO-PI with other instruments'. In: Buss, D. M. and Cantor, N. (Eds), Personality Psychology. Recent Trends and Emerging Directions, pp. 237-245, Springer, New York.

Mischel, W. (1968). Personality and Assessment, Wiley, New York.

Murray, H. A. (1938). Explorations in Personality, Oxford University Press, New York.

Newcomb, T. M. (1929). Consistency of Certain Extravert-Introvert Behavior Patterns in 51 Problem Boys, Columbia University, Teachers College, Bureau of Publications, New York.

Norman, T. (1967). 2,800 Personality Trait Descriptors: Normative Operating Characteristics for a University Population, Department of Psychology, University of Michigan, MI.

Olweus, D. (1980). 'The consistency issue in personality psychology revisited-with special reference to aggression', British Journal of Social and Clinical Psychology, 19: 377-390.

Ostendorf, F., Angleitner, A. and Ruch, W. (1986). Die Multitrait-Multimethod Analyse. Konvergente und diskriminante Validität der Personality Research Form [The MultitraitMultimethod Analysis. Convergent and Discriminant Validity of the PRF], Hogrefe, Göttingen, FRG.

Shweder, R. A. (1977). 'Likeness and likelihood in everyday thought: magical thinking in judgements about personality', Current Anthropology, 18: 637-658.

Shweder, R. A. and D'Andrade, R. G. (1980). 'The systematic distortion hypothesis'. In: Shweder, R. A. (Ed), Fallible Judgement in Behavioural Research. New Directions for the Methodology of Social and Behavioural Science, Vol. 4, pp. 37-58, Jossey-Bass, San Francisco, CA.

Strelau, J. (1987). 'The concept of temperament in personality research', European Journal of Personality, 1: 107-117.

Strelau, J. (1991). 'Are psychophysiological scores good candidates for diagnosing temperament/personality traits and for a demonstration of the construct validity of psychometrically measured traits?', European Journal of Personality, in press.

Strelau, J., Angleitner, A., Bantelmann, J. and Ruch, W. (1990). 'The Strelau Temperament Inventory Revised (STI-R): theoretical considerations and scale development', European Journal of Personality, 4: 209-235.

Thomae, H. (1960). Der Mensch in der Entscheidung [Man in his Deciding], J. A. Barth, München, FRG.

Wright, J. C. and Mischel, W. (1987). 'A conditional approach to dispositional constructs: the local predictability of social behavior', Journal of Personality and Social Psychology, 53: 1159-1177. 